\title{
Nouvelles tendances de l'enseignement supérieur chinois
}

Entretien avec le Professeur Kechao Xing

New trends in Chinese higher education

Nuevas tendencias en la educación superior en China

Pierre-Louis Gauthier

\section{OpenEdition}

Journals

Édition électronique

URL : http://journals.openedition.org/ries/2397

DOl : $10.4000 /$ ries.2397

ISSN : 2261-4265

Éditeur

Centre international d'études pédagogiques

Édition imprimée

Date de publication : 1 octobre 2000

Pagination : 123-129

ISSN : 1254-4590

Référence électronique

Pierre-Louis Gauthier, « Nouvelles tendances de l'enseignement supérieur chinois », Revue

internationale d'éducation de Sèvres [En ligne], 27 | 2000, mis en ligne le 01 octobre 2003, consulté le 30 avril 2019. URL : http://journals.openedition.org/ries/2397 ; DOI : 10.4000/ries.2397 


\section{Nouvelles tendances de l'enseignement supérieur chinois}

Entretien avec le Professeur Kechao Xing

Propos recueillis par Pierre-Louis Gauthier

\section{Résumé}

La Chine s'est engagée dans le développement qualitatif et quantitatif de son enseignement supérieur. Le recrutement, les cursus, les modes de gestion font actuellement l'objet de profonds changements, en dépit de la tradition mandarinale persistante et des disparités de cet immense pays. L'apparition d'espaces d'autonomie dans l'enseignement supérieur auprès de la traditionnelle centralisation politique et administrative illustre la souplesse d'adaptation du système chinois à une nouvelle conjoncture économique et sociale.

\section{New trends in Chinese higher education}

China has embarked on a project of qualitative and quantitative development of its higher education system. Profound changes are currently being made to recruitment, courses and administrative methods despite the enduring mandarin tradition and the disparities that exist in this vast country. The ability of the Chinese education system to adapt to a new economic and social context is illustrated by the appearance of autonomous areas in higher education alongside the traditional centralization of political and administrative institutions.

\section{Nuevas tendencias en la educación superior en China}

China se ha lanzado en una fase de desarrollo cualitativo y cuantitativo de su enseñanza superior. La contratación, los estudios universitarios, las modalidades de gestión experimentan cambios profundos, a pesar de la tradición mandarinal persistente y de las disparidades de este inmenso país. La emergencia de espacios autónomos en la enseñanza superior al lado de la tradicional centralización política y administrativa muestra la capacidad de adaptación del sistema chino a una nueva coyuntura económica y social. 
Toute réforme de l'enseignement en Chine entraîne des conséquences qui dépassent, par leur ampleur et leur résonance à moyen terme, toute démarche équivalente en Europe. Lentes à mettre en œuvre compte tenu de l'immensité de la tâche à accomplir, les réformes, une fois mises en route, se prolongent dans le temps entraînant parfois des effets imprévisibles.

Dans un précédent article ${ }^{1}$, les tendances actuelles de l'évolution de l'enseignement supérieur chinois avaient été succinctement abordées. Ces tendances restent caractérisées par les points suivants : moins d'universités, moins de centralisation, plus de coopération verticale et horizontale entre universités.

L'entretien qui suit porte sur le devenir de ces tendances au seuil du XXIe siècle et les nouvelles perspectives qui se dégagent.

Pierre-Louis Gauthier - Quelle est actuellement la situation générale de l'enseignement supérieur en Chine?

Kechao Xing - L'enseignement supérieur chinois connaît un développement continu tant sur le plan quantitatif que sur le plan qualitatif. En une décennie, le nombre d'étudiants inscrits dans les établissements d'enseignement supérieur est ainsi passé de 600000 à 2,5 millions en 1999, répartis dans un millier d'institutions ${ }^{2}$.

Le nombre de grades universitaires délivrés chaque année a crû dans les mêmes proportions : pendant la période 1991-1999, le nombre de diplômés est passé de 600000 à 850000 , le nombre de master degree délivrés a doublé tandis que le nombre de doctorats a quintuplé. De façon concomitante, la demande sociale et économique adressée à l'université par la société civile s'est accrue. Le nombre d'inscrits à l'examen d'entrée au $3^{\mathrm{e}}$ cycle a triplé en dix ans : ce renforcement est un signe prometteur.

Pourtant, en dépit de cette progression, le taux de scolarisation dans l'enseignement supérieur reste modeste. Il faudra certes attendre les résultats du recensement de la population commencé début octobre 2000 pour obtenir une approche plus fine. Mais on peut estimer que ce taux ne dépasse pas actuellement 700 étudiants pour 100000 actifs, ce qui place la Chine certes devant l'ensemble des pays africains, par exemple (100 en moyenne), mais loin derrière l'ensemble des pays développés (moyenne : 2 500, France : 3000 ) et très loin derrière les pays d'Amérique du Nord $(5000)$. La progression du nombre d'étudiants est toutefois en hausse constante pour la Chine, contrairement à la plupart des pays européens qui voient ce nombre fléchir du fait de l'affaissement démographique de leurs populations (pour la France, la diminution du nombre

1 XING Kechao, GAUTHIER Pierre-Louis, "Regards croisés sur l'éducation en Chine ", Revue internationale d'éducation - Sèvres, $n^{\circ} 15$, septembre 1997.

2 World Data on Education, UNESCO, Paris, 1999. 
d'étudiants, de l'ordre de $-2 \%$ par an, devrait être partiellement compensée par le développement des filières technologiques).

La croissance s'est accompagnée d'une amélioration sensible de la qualité des enseignants. Les mesures de régulation pour la délivrance des diplômes de l'enseignement supérieur, prises en 1993 par la Commission d'État pour l'éducation, commencent à produire leurs effets. Ainsi, le pourcentage des docteurs a augmenté dans des proportions importantes, passant de $1 \%$ en 1991 à $5 \%$ en 1999. En moyenne, on compte 100000 nouveaux diplômés post-licence par an ${ }^{3}$. Il est de plus en plus rare de rencontrer, dans l'enseignement supérieur, un enseignant qui ne soit pas, au minimum, engagé dans le troisième cycle. Les fortes disparités entre établissements (universités, instituts, écoles supérieures) qui avaient été décrites précédemment se sont donc atténuées.

Pour garantir et améliorer ce niveau de qualité, les enseignants sont soumis à une évaluation annuelle de plus en plus rigoureuse de leurs prestations en matière d'enseignement (nombre d'heures effectives) et de recherche (communications, articles, ouvrages). Les projets de recherche sont pris en compte au niveau de l'établissement, de la province ou du pays, et viennent s'ajouter au curriculum vitae de chaque enseignant.

P.-L. G. - Des conditions matérielles médiocres ont longtemps pesé sur le fonctionnement de l'enseignement supérieur chinois : qu'en est-il aujourd'hui?

K. X. - Après de longues années difficiles, les enseignants ont vu, à partir des années quatre-vingt-dix, leur situation professionnelle, de même que leurs conditions d'existence, s'améliorer sensiblement. Même si les salaires des enseignants restent modestes, leur revalorisation est une réalité concrète (de l'ordre de $100 \%$ ). Mais dans le même temps, l'éventail des salaires s'est élargi soulignant davantage les différences entre les grades.

Les conditions d'exercice connaissent également des progrès importants et concrets. On voit partout sur les campus surgir de nouveaux bâtiments pour la recherche, les bibliothèques, les logements. L'équipement en nouvelles technologies d'information a été rapidement mené dans tous les établissements. Ces créations tendent à sortir définitivement l'enseignement supérieur chinois d'un certain sous-développement. Certes de nombreux retards subsistent et on ne citera que les conditions d'hébergement des étudiants et des jeunes enseignants qui restent souvent très médiocres.

P.-L. G. - L'enseignement supérieur chinois reste-t-il soumis au centralisme?

K. X. - Le lecteur se souvient peut-être que dans l'article déjà cité ${ }^{4}$, sous le sous-titre "Une, deux, trois Chine ", on insistait sur les disparités régionales, déjà anciennes en Chine, notamment entre les provinces de l'Est et celles

3 En France, ce nombre s'établit pour 1999 à 60175 diplômés (DEA, DESS, doctorat).

4 Ibid, note 1. 
de l'Ouest. Il faudra, ici et maintenant, mettre à mal un cliché fortement ancré dans les esprits occidentaux, d'une «Chine impériale ", gouvernant de manière uniforme tous les domaines d'activité.

En fait, les trente-et-une entités territoriales (4 villes ou « municipalités ${ }^{5}, 5$ régions autonomes des minorités, 22 provinces) ont reçu une large autonomie dans le domaine économique dès les années quatre-vingt, à un moment où l'on commençait à concilier économie socialiste planifiée et économie de marché. $\mathrm{Au}$ cours des années suivantes et jusqu'à maintenant, cette réorientation politique, en s'accentuant, a mis en évidence l'inadaptation du cadre juridique centré sur l'entreprise d'État, " unité de travail " responsable tout à la fois non seulement de la production, mais également des domaines de la santé, des pensions, du logement, du chômage, de l'éducation enfin. Ensuite, par simple conséquence structurelle, cette autonomie s'est progressivement élargie à d'autres secteurs, dont celui de l'éducation, tout en s'efforçant de maintenir un équilibre entre les prérogatives et les ressources du pouvoir central et des provinces. Les réformes qui se sont succédées jusqu'en 2000 ont permis à bon nombre de provinces, suffisamment pourvues de ressources locales, de définir des stratégies de développement autonomes et tout à fait indépendantes du pouvoir central.

L'enseignement supérieur a su bénéficier de cette évolution vers plus d'autonomie. L'autonomie s'est traduite tout d'abord par un changement structurel de la gestion financière et administrative. Le gouvernement et ses ministères se sont déchargés de nombreuses responsabilités au profit des entités territoriales, municipalités, villes, régions ou provinces. Le changement de politique s'opère par transfert de crédits affectés, mais le plus souvent par de nouvelles ressources financières mises à la disposition de l'échelon territorial (droits d'inscription, impôts, taxes, etc.). Ce mouvement touche à présent presque $40 \%$ des établissements d'enseignement supérieur chinois: en dix années, plus de quatre cents établissements "nationaux » ou " ministériels ", sur un millier, sont devenus " provinciaux " ou " municipaux " dans le cadre de ce mouvement de décentralisation.

P.-L. G. - Pouvez-vous caractériser ce mouvement de décentralisation? Quels en sont sur l'enseignement supérieur les effets positifs ou plus contestables?

K. X. - Il faut savoir que le mouvement de décentralisation s'accompagne simultanément d'un regroupement des établissements au niveau local. L'objectif du regroupement est d'obtenir une optimisation des différents secteurs de la gestion humaine, financière, matérielle. Au cours des dix dernières années, plus de six cents établissements ont participé à de tels regroupements aboutissant en définitive à deux cent cinquante structures nouvelles. Par exemple, les quatre universités de Hanzhou, représentant environ 20000 étudiants, sont désormais regroupées en un seul établissement.

5 II s'agit en fait de régions centrées sur des villes multimillionnaires, comme Beijing ou Shanghaï. 
Au niveau national, en se déchargeant de la globalité de ses responsabilités, le ministère de l'Éducation peut se consacrer à la gestion de quelques universités de prestige qui sont pour le moment au nombre de cent dix (au lieu de plus de deux cents quelques années auparavant). Au niveau provincial, la gestion s'effectue avec plus de ressources que précédemment, s'adaptant plus souplement aux besoins sociaux et économiques réels. Le regroupement favorise la concentration des potentialités et des moyens intellectuels et matériels en vue d'une meilleure économie et d'un meilleur rendement, tout en rendant l'établissement plus «fort ».

L'optimisation des moyens de gestion, obtenue grâce au regroupement, se traduit pour les présidents et les exécutifs des établissements par une recentration de leurs tâches. Ils peuvent mieux et davantage se consacrer à l'enseignement et à la recherche, laissant à d'autres services de gestion spécialisés, les problèmes de logistique courante (restaurant, hébergement, entretien, réparation, voire même construction).

Le paradoxe de cette évolution vers une forte concentration des pouvoirs et des moyens, au profit d'un nombre réduit d'établissements, est que chacun d'entre eux se trouve investi de plus d'autonomie. Ainsi, l'examen d'entrée à l'université se déroulait jusque-là selon un rite quasi immuable, à des dates nationales traditionnellement fixées aux 7,8 et 9 juillet de chaque année. Il était fondé sur cinq épreuves obligatoires, à savoir, pour les littéraires, le chinois, les mathématiques, l'anglais, l'histoire et la politique, les deux dernières disciplines étant remplacées, pour les scientifiques, par la physique et la chimie. L'examen actuel se transforme, à titre expérimental, en un système à trois épreuves obligatoires (chinois, mathématiques, anglais), plus $n$ matières laissées au choix de l'établissement. De plus, le calendrier des épreuves est étalé dans le temps de manière à laisser aux candidats la possibilité de se présenter en différents lieux.

Autre exemple, les droits d'inscription, fixés dans une marge nationale sont désormais perçus directement par l'établissement en vue d'une gestion plus autonome. Les droits d'inscription représentent une source de revenus non négligeable pour l'établissement. La participation des étudiants aux coûts de l'enseignement varie considérablement d'un pays à l'autre. On sait qu'elle n'est pas fonction du niveau de vie du pays concerné (États-Unis : $25 \%$; France : $2 \%$ ). En moyenne, en Chine, ces droits permettent de répondre à $9 \%$ des dépenses récurrentes de l'établissement. Précisons que les droits d'inscription sont modulables selon les résultats obtenus aux examens d'entrée. Selon le rang obtenu, les droits peuvent varier de 1 à 10 . En cas d'échec, on peut même, dans certains cas, racheter financièrement l'admission. On touche ici au financement global de l'enseignement supérieur qui atteint en Chine 15,6\% du budget général de l'éducation (France : 14,51\%). Le budget est réparti suivant le nombre d'étudiants diplômés de l'année antérieure. Ce système signifie que les résultats obtenus par l'établissement ont une incidence quasi-immédiate sur les ressources. 
Ces différentes tendances au sein de l'enseignement supérieur chinois, apparues dès les années quatre-vingt-dix, relèvent d'un certain pragmatisme. Elles révèlent la distance prise par rapport à l'ancien dirigisme centralisateur. Elles comportent certes des aspects positifs, mais aussi certains inconvénients.

$\mathrm{Au}$ nom de l'autonomie récemment acquise, certains établissements ouvrent de nouveaux départements, parfois étrangers à leur vocation première. Ainsi, l'université Beida de Beijing a ouvert, en 2000, un institut de sciences de l'éducation regroupant plusieurs départements et centres de recherche dans ce domaine. Pourtant cette université, la première en Chine historiquement et académiquement parlant, n'a jamais eu pour vocation la formation des enseignants. Par contre, l'université normale La Capitale, toujours à Beijing, qui a bien pour vocation première la formation des enseignants, a créé au sein du département d'histoire, une section de "gestion du tourisme" !

Les limites de l'autonomie sont donc à redéfinir sur un territoire donné à l'aide de critères de cohérence, de compétences et de complémentarité. La même problématique se retrouve tant à l'échelle provinciale que nationale.

En revanche, lorsque la situation n'est pas mûre, le regroupement émanant d'une décision prise au niveau central n'emporte pas nécessairement l'adhésion de l'ensemble de l'établissement. On constate alors un manque d'implication dans la mise en œuvre, voire même de l'inertie ou de l'hostilité, ce qui réduit d'autant l'effet attendu du regroupement.

Le regroupement a par ailleurs pour conséquence immédiate, de renforcer les plus forts par concentration de leurs moyens déjà considérables, notamment pour la recherche, augmentant encore le prestige de quelques universités de renom. Inversement, les établissements plus faibles, notamment dans les régions défavorisées ${ }^{6}$, et avec lesquels on ne souhaite pas coopérer, se retrouvent par la force des choses affaiblis. On voit bien que les "règles " de l'économie de marché ne sont pas loin.

Cette évolution vers la décentralisation tend à accroître les disparités entre établissements et régions dans une même province. Ainsi l'examen d'entrée "régionalisé " n'offre plus le même degré d'exigence d'un établissement à l'autre. Un classement officieux des établissements se fait jour inévitablement, alors que le diplôme est réputé être de valeur identique. Il manque actuellement un système d'évaluation global du système de l'enseignement supérieur, aboutissant à un étalonnage des différents secteurs d'enseignement et de recherche et permettant un pilotage national. La création d'instances d'évaluation dans les systèmes éducatifs européens soulève beaucoup d'intérêt chez les responsables chinois ${ }^{7}$.

6 Ibid, note 1.

7 Cf "L'évaluation des systèmes éducatifs", Revue internationale d'éducation - Sèvres, $n^{\circ}$ 26, juin 2000. 
L'échelle des revenus des enseignants reflète également de nombreuses disparités. Parmi ces contradictions, le décalage croissant des revenus entre les différents grades et groupes d'âge des enseignants entraîne parfois découragement et désaffection chez les plus jeunes. Il faudra trouver rapidement un nouveau mécanisme de rémunération stimulant davantage les catégories plus défavorisées et notamment les jeunes chercheurs et les grades inférieurs.

Le mouvement de décentralisation qui s'est emparé de l'enseignement supérieur chinois, tous établissements confondus, correspond certes aux nécessités de l'évolution sociale et économique de cette fin du xxe siècle. Dans le cadre de la vaste et originale révolution industrielle que connait la Chine, face à l'initiative économique, l'enseignement supérieur ne pouvait que suivre, sinon devancer, et s'adapter localement.

Le nouveau siècle s'ouvre sur la mondialisation qui peut remettre en cause un équilibre institutionnel encore fragile, une évolution encore incomplète. Pour pallier ces risques majeurs, la Chine ne pourra plus désormais échapper aux réformes permanentes, ni au recours périodique à l'intervention du pouvoir central tutélaire. 